\title{
Simultaneous squamous cell carcinoma with primary malignant fibrous histiocytoma of the larynx: A case report
}

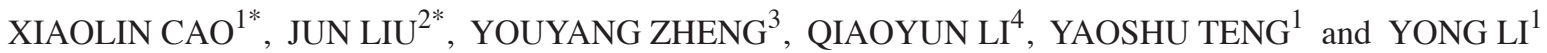 \\ ${ }^{1}$ Department of Otorhinolaryngology - Head and Neck Surgery, Hangzhou First People's Hospital, Hangzhou, Zhejiang; \\ ${ }^{2}$ Department of Otorhinolaryngology, Qilu Hospital of Shandong University, Jinan, Shandong; \\ ${ }^{3}$ Department of Oral Medicine, Second Affiliated Hospital, Zhejiang University School of Medicine; \\ ${ }^{4}$ Department of Pathology, Hangzhou First People's Hospital, Hangzhou, Zhejiang, P.R. China
}

Received November 2, 2011; Accepted January 19, 2012

DOI: $10.3892 / \mathrm{mmr} .2012 .775$

\begin{abstract}
Simultaneous multiple malignancies of the larynx are rarely reported. In this study, we describe a case with simultaneous laryngeal, moderately differentiated squamous cell carcinoma (SCC) and primary malignant fibrous histiocytoma (MFH) in a patient presenting with progressive hoarseness and without cervical lymphadenopathy. The clinical presentation, intraoperative findings, radiographic images and pathology slides are presented. The diagnosis was confirmed using H\&E staining and immunohistochemical testing. A partial laryngectomy with bilateral neck selective dissection was performed. The patient survived for more than 46 months following surgery without recurrence or metastasis. To our knowledge, this is the first report of a case with simultaneous laryngeal SCC and primary MFH in the English literature. The results indicate that the markers used to assess the prognosis of $\mathrm{MFH}$ may also be used to assess simultaneous laryngeal SCC and primary $\mathrm{MFH}$, and that laryngectomy to preserve function may be performed in early-stage patients.
\end{abstract}

\section{Introduction}

In 1889 , the first case of multiple primary tumors was reported by Billroth (1). In 1932, Warren and Gates (2) established the diagnostic criteria for multiple primary tumors, which are still used today. Simultaneous multiple primary malignancies of the larynx are very rare. To our knowledge, only six cases with

Correspondence to: Dr Yong Li, Department of Otorhinolaryngology - Head and Neck Surgery, Hangzhou First People's Hospital, 261 Huansha Road, Hangzhou, Zhejiang 310006, P.R. China

E-mail:xiaolinz@sohu.com

*Contributed equally

Abbreviations: SCC, squamous cell carcinoma; MFH, malignant fibrous histiocytoma

Key words: squamous cell carcinoma, malignant fibrous histiocytoma, larynx simultaneous multiple primary malignancies of the larynx have been reported to date. In this study, we report a case of simultaneous laryngeal squamous cell carcinoma (SCC) and primary malignant fibrous histiocytoma (MFH).

\section{Case report}

The present study was approved by the Institutional Review Board of the Hangzhou First People's Hospital, Zhejiang Province, China. A 69-year-old Chinese woman, with no history of smoking or alcohol consumption, was referred to our hospital in August 2007. She complained of hoarseness for more than one year, becoming progressively during the last two months prior to presentation, without other symptoms. Significant physical findings were limited to the head and neck. Indirect and flexible electronic laryngoscopy revealed a $1.5 \times 1.0 \times 1.0 \mathrm{~cm}$ mass derived from the subglottic region (under the anterior commissure) and the involvement of the anterior commissure. The activity of the bilateral vocal cord was symmetrically limited. The surface of the mass was smooth without ulcers, erosion or hemorrhaging. The CT scans showed the presence of a subglottic mass under the anterior commissure and no pathological swollen lymph nodes (Fig. 1a). The results of neck and abdominal ultrasonography, chest X-ray and laboratory tests were normal. The patient had a ten-year plus history of hypertension and diabetes mellitus and her blood pressure and blood glucose were well controlled using medication. No radiotherapy, surgery or trauma to the neck was reported prior to onset. Biopsy was performed prior to surgery.

A fronto-lateral partial laryngectomy with bilateral arytenoid preservation under general anesthesia with safety margins $(>10 \mathrm{~mm})$, coupled with a bilateral neck selective dissection, were performed. The mass was removed successfully and the defection resulting from partial laryngectomy was reconstructed with bilateral sternohyoid muscles. The excised tumor was confirmed as moderately differentiated SCC and MFH using H\&E staining (Fig. 1b-d) and immunohistochemical pathological testing (Fig. 2a-d). Table I lists the results of the immunohistochemical testing of the tumor. The final pathological diagnosis was moderately differentiated SCC with MFH, all margins of the resected tumors appeared to be free of disease and no metastatic lymph nodes were found in 


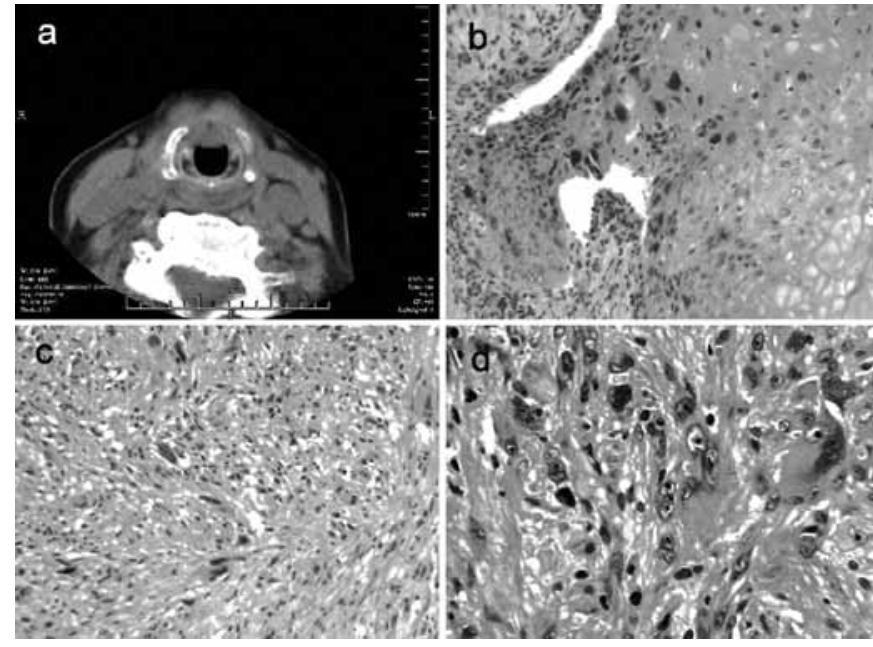

Figure 1. (a) Axial CT plain scan demonstrating the mass located in the fron of the larynx and under the anterior commissure; (b) moderately differentiated SCC, H\&E (magnification, x100); (c) MFH, H\&E (magnification, x100); (d) MFH, H\&E (magnification, x200). SCC, squamous cell carcinoma; MFH, malignant fibrous histiocytoma.

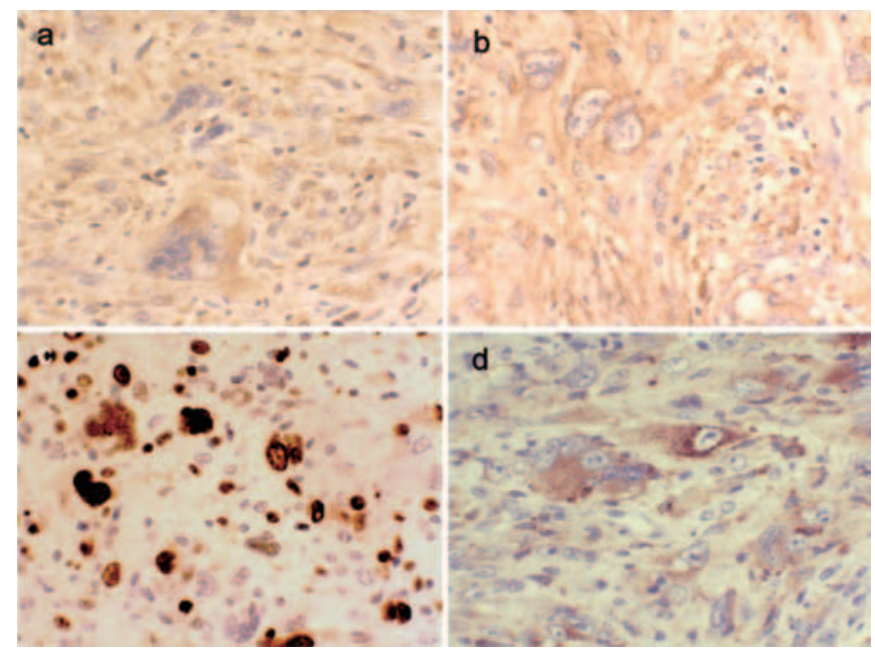

Figure 2. Immunohistochemical staining in MFH of the larynx. (a) Vimentin; (b) SMA; (c) Ki-67; (d) CD68 (magnification, x200). MFH, malignant fibrous histiocytoma. SMA, smooth muscle actin.

the excised neck tissue. The follow-up period was more than 46 months, and the patient survived without recurrence and metastasis.

\section{Discussion}

Over $95 \%$ of laryngeal malignant neoplasms are SSCs which are derived from mucous epithelium of the larynx. Other tumors, including adenocarcinoma, undifferentiated carcinoma and lymphosarcoma, are rare. MFH, a subtype of sarcoma, is a type of non-epithelial tumor that may be of mesenchymal origin and is composed of various cell types, including fibroblasts, histiocyte-like cells and atypical giant cells. The incidence of MFH is reported to be less than $2 \%$ of all malignancies in the larynx and accounts for approximately $5 \%$ of all sarcomas of the head and neck.
Table I. Results of immunohistochemical analysis.

\begin{tabular}{lc}
\hline Antibody & Result \\
\hline Cytokeratins AE1/AE3 & - \\
Epithelial membrane antigen & - \\
Desmin & - \\
Vimentin & + \\
Smooth muscle actin & + \\
S-100 protein & - \\
Ki-67:30 & + \\
CD68 & + (sporadic) \\
MyD1 & - \\
\hline
\end{tabular}

Although the etiology of MFH remains unclear, it is generally accepted that radiation may induce MFH (3). According to the diagnostic criteria proposed by Cahan et al (4) and Bradley (5), the majority of cases with MFH of the larynx are associated with radiation (6). Our patient denied history of treatment with or exposure to radiation and had no other observed pathogenic factors, indicating that the etiology of MFH requires further investigation. The prognosis of cases with $\mathrm{MFH}$ is associated with tumor size, age, gender and histological grade. Tumor size less than $3 \mathrm{~cm}$, age over 60 years, female gender and lowgrade histology are regarded as favorable prognostic factors for MFH (7). The patient in our study was a 69-year-old woman, with tumor size smaller than $3 \mathrm{~cm}$, indicating favorable prognosis. In this case, the patient survived without tumors for more than three years following surgery. These results are consistent with those of previous studies and suggest that the markers for evaluating the prognosis of MFH may also be used in patients with laryngeal SCC with simultaneous primary MFH.

The incidence of primary multiple malignant tumors in the larynx is exceedingly low. The diagnostic criteria of multiple primary tumors, which remain in use to date, were proposed by Warren and Gates (2) in 1932. We reviewed the previous literature and found that all six cases were male patients with a history of tobacco and alcohol consumption. With the exception of one case with chondrosarcoma and epidemoid carcinoma, these cases comprised simultaneous SCC and adenocarcinoma (two cases), chondrosarcoma (one case), leiomyosarcoma (one case), verrucous carcinoma and squamous papilloma (one case). To our knowledge, this is the first report of a case with simultaneous SCC and primary MFH. In cases with multiple tumors of the larynx, treatment is carried out according to the types of tumor involved. For well- and moderately-differentiated SCC, surgery is the first treatment considered. For MFH, simple radiotherapy or simple chemotherapy is not recommended, whereas surgery or comprehensive therapy combined with surgery is regarded as the best choice. Sabesan et al (8) suggested that radical resection of a tumor is a more efficacious method for improving survival and reducing recurrence. However, partial laryngectomy (9) and laryngomicrosurgery (10), which preserve laryngeal function and improve quality of life following surgery, are recommended in early MFH. Since the tumor in our patient was small and localized to one region, partial laryngectomy with bilateral neck dissection was performed without radiotherapy/ 
chemotherapy (pre- or post-operation). During the follow-up period, no evidence was found of local recurrence, cervical lymph nodes or distant metastasis. Therefore, laryngectomy with preservation of laryngeal function can be performed in many patients with early-stage tumors.

In conclusion, simultaneous multiple malignancies of the larynx are rare. In this study, we report a unique case with laryngeal moderately differentiated SCC and simultaneous primary MFH, which was confirmed by H\&E staining and immunohistochemical testing. Our results show that the markers used to assess the prognosis of MFH may also be used to assess simultaneous laryngeal SCC and primary MFH and that, to preserve laryngeal function, laryngectomy can be performed in patients with early-stage tumors.

\section{References}

1. Billroth T: Die allgemeine chirurgische pathologie und therapie In: 51 Vorlesungen: Ein Handbuch Fur Studirende und Arzte. 14th edition. Reimer G, Berlin, pp908, 1889.

2. Warren S and Gates O: Mutiple primary malignant tumors: a survey of the literature and a statistical study. Am J Cancer 16 $1358-1414,1932$.
3. Kirova YM, Feuilhade F, Calitchi E, et a1: Radiation-induced sarcoma after breast cancer. Apropos of 8 cases and review of the literature. Cancer Radiother 2: 381-2386, 1998.

4. Cahan WG, Woodard HQ, Higinbotham NL, Stewart FW and Cloey BL: Sarcoma arising in irradiated bone: report of eleven cases. Cancer 82: 8-34, 1998.

5. Bradley PJ: Radiation-induced tumors of the head and neck. Curr Op Otolaryngol Head Neck Surg 10: 97-103, 2002.

6. Guney E, Yigitbasi OG, Balkanli S and Canoz OM: Postirradiation malignant fibrous histiocytoma of the larynx: a case report. Am J Otolaryngol 23: 293-296, 2002.

7. Haberal I, Samim E, Astarci M and Ozeri C: Radiation-induced malignant fibrous histiocytoma of the neck in a patient with laryngeal carcinoma. Am J Otolaryngol 22: 146-149, 2001.

8. Sabesan T, Xuexi W, Yongfa Q, Pingzhang T and Ilankovan V: Malignant fibrous histiocytoma: outcome of tumours in the head and neck compared with those in the trunk and extremities. Br J Oral Maxillofac Surg 44: 209-212, 2006.

9. Soh KB, Westmore GA, Moir AA and Colloby PS: Malignant fibrous histiocytomas of the larynx - report of two cases. Ann Acad Med Singapore 25: 878-881, 1996.

10. Kuwabara H, Saito K, Shibanushi T and Kawahara T: Malignant fibrous histiocytoma of the larynx. Eur Arch Otorhinolaryngol 251: 178-182, 1994. 